\title{
Salt-sensitive hypertension is associated with dysfunctional Cyp4a10 gene and kidney epithelial sodium channel
}

\author{
Kiyoshi Nakagawa, ${ }^{1}$ Vijaykumar R. Holla, ${ }^{1}$ Yuan Wei, ${ }^{2}$ Wen-Hui Wang, ${ }^{2}$ \\ Arnaldo Gatica, ${ }^{1}$ Shouzou Wei, ${ }^{1}$ Shaojun Mei, ${ }^{1}$ Crystal M. Miller, ${ }^{1}$ Dae Ryong Cha, ${ }^{1}$ \\ Edward Price Jr., ${ }^{3}$ Roy Zent, ${ }^{1,4}$ Ambra Pozzi, ${ }^{1}$ Matthew D. Breyer, ${ }^{1}$ Youfei Guan, ${ }^{1}$ \\ John R. Falck, ${ }^{5}$ Michael R. Waterman, ${ }^{3}$ and Jorge H. Capdevila ${ }^{1,3}$

\begin{abstract}
${ }^{1}$ Department of Medicine, Vanderbilt University, Nashville, Tennessee, USA. ${ }^{2}$ Department of Pharmacology, New York Medical College, Valhalla, New York, USA. ${ }^{3}$ Department of Biochemistry, Vanderbilt University, Nashville, Tennessee, USA. ${ }^{4}$ Veterans Affairs Hospital, Nashville, Tennessee, USA. ${ }^{5}$ Department of Biochemistry, University of Texas Southwestern Medical Center, Dallas, Texas, USA.
\end{abstract}

\begin{abstract}
Functional and biochemical data have suggested a role for the cytochrome $\mathbf{P 4 5 0}$ arachidonate monooxygenases in the pathophysiology of hypertension, a leading cause of cardiovascular, cerebral, and renal morbidity and mortality. We show here that disruption of the murine cytochrome P450, family 4, subfamily a, polypeptide 10 (Cyp4a10) gene causes a type of hypertension that is, like most human hypertension, dietary salt sensitive. Cyp4a10 $10^{-/}$mice fed low-salt diets were normotensive but became hypertensive when fed normal or high-salt diets. Hypertensive Cyp4a10 ${ }^{-/-}$mice had a dysfunctional kidney epithelial sodium channel and became normotensive when administered amiloride, a selective inhibitor of this sodium channel. These studies (a) establish a physiological role for the arachidonate monooxygenases in renal sodium reabsorption and blood pressure regulation, (b) demonstrate that a dysfunctional Cyp4a10 gene causes alterations in the gating activity of the kidney epithelial sodium channel, and (c) identify a conceptually novel approach for studies of the molecular basis of human hypertension. It is expected that these results could lead to new strategies for the early diagnosis and clinical management of this devastating disease.
\end{abstract}

\section{Introduction}

Prevalence, complexity, and multiple medical and socioeconomic consequences make hypertension a major health challenge for most of the Western world (1). While environmental factors and coexisting conditions play a role in the development and progression of hypertension, segregation and linkage analyses indicate that multiple genetic factors contribute to its complex etiology (2-7). Furthermore, clinical studies show that the cardiovascular and renal morbidity and mortality resulting from hypertension are markedly reduced by timely diagnosis and early clinical intervention (1). As the kidneys play a central role in the control of body salt and fluid balance, they are frequent targets for the treatment of hypertension, especially those forms sensitive to dietary salt (2-5). However, since the molecular basis of prevalent forms of the disease remains uncertain, its early diagnosis and treatment are largely symptomatic. It is expected that the identification of novel pathways/genes involved in blood pressure variations $(3,6,7)$ will lead to new therapeutic targets and to improved diagnosis and prevention. Indeed, early detection and treatment are urgently needed to prevent the dangerous and profound consequences of untreated chronic hypertension.

The metabolism of endogenous arachidonic acid (AA) to epoxyeicosatrienoic acids (EETs) and 20-hydroxyeicosatetraenoic acid

Nonstandard abbreviations used: AA, arachidonic acid; CD, collecting duct; Cyp4a10, cytochrome P450, family 4, subfamily a, polypeptide 10; DHET, dihydroxyeicosatrienoic acid; EET, epoxyeicosatrienoic acid; ENaC, epithelial sodium channel; 20-HETE, 20-hydroxyeicosatetraenoic acid; LC/MS/MS, liquid chromatographytandem mass spectroscopy; NPo, channel number multiplied by the open probability; P450, cytochrome P450; Wy, Wyeth 14643.

Conflict of interest: The authors have declared that no conflict of interest exists. Citation for this article: J. Clin. Invest. 116:1696-1702 (2006). doi:10.1172/JCI27546
(20-HETE) by the epoxygenase and $\omega$-hydroxylase branches of the cytochrome P450 (P450) AA monooxygenase is well established $(8,9)$ as are the biological activities of these metabolites (9-11). Earlier studies identified members of the P450 CYP2C and CYP4A gene subfamilies as functionally relevant AA epoxygenases and $\omega$-hydroxylases, respectively (8-11), and implicated them in the control of renal function and blood pressure (9-11). However, the unequivocal interpretation of these studies is complicated by the in vitro nature of the functional data available (8-11) and genetic complexity of the animal models studied (9-13). Consequently, notwithstanding their potential clinical importance, the physiological role of these enzymes in blood pressure regulation remains ambiguous and in need of experimental confirmation. We show here that a dysfunctional cytochrome P450, family 4, subfamily a, polypeptide 10 (Cyp4a10) gene causes a type of hypertension that is dietary salt sensitive and associated with alterations in the activity of the renal epithelial sodium channel (ENaC).

\section{Results}

Disruption of the Cyp4a10 gene causes bypertension. Mating and selection of germ line chimeras carrying a mutant Cyp4a10 allele were

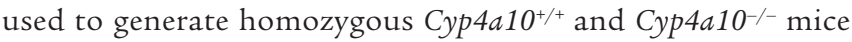
(from the progeny of an F2 cross of Cyp $4 a 10^{+/-}$heterozygous mice) in isogenic $129 / \mathrm{SvJ}$ backgrounds. Cyp $4 a 10^{-/-}$mice developed normally and lacked outward symptoms of disease or organ malformation. Light microscopy analysis of paraffin-embedded kidney sections and analysis of renal function by measurement of plasma creatinine levels by HPLC (14) demonstrated that disruption of the Cyp4a10 gene had little or no effect on organ morphology (not shown) or renal function before or after animal salt loading $(157 \pm 7$ and $168 \pm 10 ; 161 \pm 6$ and $169 \pm 9 \mu \mathrm{g}$ 
Table 1

Male and female Cyp4a10-- mice are hypertensive

$\begin{array}{lcc} & \text { Cyp4a10+/+ } & \text { Cyp4a10 }^{+/-} \\ \text {Females } & 103 \pm 3 & 147 \pm 3^{\mathrm{A}} \\ \text { Males } & 105 \pm 2 & 151 \pm 3^{\mathrm{A}}\end{array}$

The systolic blood pressure (in $\mathrm{mmHg}$ ) of conscious 12- to 14-week-old male and female mice fed solid diets containing $0.3 \% \mathrm{NaCl}(\mathrm{w} / \mathrm{w})$ was measured by the tail cuff method. Shown are averages \pm SEM calculated from groups of $15 \mathrm{Cyp} 4 \mathrm{a} 10^{-/-}$males, $10 \mathrm{Cyp} 4 \mathrm{a} 10^{-/-}$females, 16

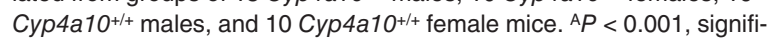
cantly different from wild type.

creatinine/dl for wild-type and Cyp4a10-/ mice fed normal or high-salt diets, respectively; $n \geq 4$ ).

Measurements of systolic blood pressure in adult Cyp4a10-/- and Cyp $4 a 10^{+/+}$mice provided decisive evidence of a role for Cyp4a10 in blood pressure control (Table 1). Compared with wild-type mice, male and female Cyp4a10-- mice showed significantly increased systolic blood pressure when measured by either the tail cuff method (Table 1) or a carotid artery catheter (15) (average increases of 27 and $22 \mathrm{mmHg}$ for the systolic blood pressure of male and female mice, respectively; $n=11 ; \mathrm{SEM} \leq 10 \%$ of the mean). Male and female Cyp4a10-- mice were equally hypertensive (Table 1 ), and their plasma androgen levels and renal 20-HETE synthase activity were comparable to those of normotensive Cyp4a10 $0^{+/+}$mice (15).

Hypertension in Cyp4a10-- mice is dietary salt sensitive. To explore the mechanism of the hypertensive phenotype, male Cyp4a10-- and Cyp4a10 $10^{+/+}$mice were fed diets containing (w/w) either $0.05 \%$ (low salt), $0.3 \%$ (normal salt), or $8 \% \mathrm{NaCl}$ (high salt) and, after 3 weeks, their systolic blood pressure, urine volume, and urine sodium and potassium concentrations were measured. While dietary salt had no significant effects on the blood pressure of wild-type animals (Figure 1A), Cyp4a10- mice on normal or high-salt diets were severely hypertensive and became normotensive when fed low-salt diets (Figure 1A). These salt-induced pressure changes were accompanied by reductions in urinary output during 16-hour collection periods (Figure 1B) and thus in the amount of sodium $(43 \% \pm 15 \% ; 44 \% \pm 7 \%$; and $64 \% \pm 5 \%$ of wildtype mice on low-salt, normal, and high-salt diets, respectively; $n \geq 14$ ) and potassium $(57 \% \pm 0.6 \% ; 52 \% \pm 0.4 \%$; and $79 \% \pm 0.6 \%$ of wild-type mice on low-salt, normal, and high-salt diets, respectively; $n \geq 14$ ) excreted during this time period. Furthermore, nuclear magnetic resonance measurements (16) showed that free fluid volumes in Cyp4a10-mice fed normal salt were higher than those of matched Cyp $4 a 10^{+/+}$ controls $(2.0 \% \pm 0.1 \%$ and $1.5 \% \pm 0.1 \%$ of body weight, respectively; wild type versus knockout, $P<0.002 ; n=8)$. Taken together, these results show that a dysfunctional Cyp4a10 gene causes reduced sodium and potassium excretion, fluid retention, and hypertension.

Disruption of the Cyp4a10 gene does not alter renal 20-HETE synthase activity. Pro- and antihypertensive roles have been described for 20-HETE and its CYP4A2 synthase (9-11), and 20-HETE has been characterized as a natriuretic eicosanoid and a renal vasoconstrictor (9-11). The phenotypic analysis of Cyp4a14-/- mice confirmed the hemodynamic properties of 20-HETE (15) and identified Cyp4a12 as an androgen-regulated prohypertensive 20-HETE synthase (15). Among the murine Cyp4a proteins $(15,17)$, only Cyp4a12 showed significant 20-HETE synthase activity (turnover: $6.7 \pm 0.6,1.0 \pm 0.3$, and $\leq 0.01 \mathrm{~min}^{-1}$ for Cyp4a12, -4a10, and -4a14, respectively; $n \geq 6$ ). Northern blots and enzymatic assays showed that disruption of the
Cyp4a10 gene had only limited effects on kidney Cyp4a12 expression (not shown) or 20-HETE synthase activity (59 \pm 3 and $54 \pm 2$

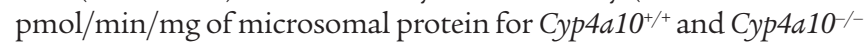
mice, respectively). These results indicated that hypertension in Cyp4a10-/- mice was unrelated to changes in kidney 20-HETE biosynthesis and its effects on renal hemodynamics (15).

Cyp4a10-/ hypertension is amiloride sensitive. Within the P450derived metabolites of AA, the EETs have attracted special attention because of their multiple vasoactive, transport, and antihypertensive properties (8-11). Thus, for example, earlier studies demonstrated that renal EET biosynthesis was sensitive to dietary salt intake (18), that inhibition of renal epoxygenase caused saltsensitive hypertension (19), and that hypertensive Dahl salt-sensitive rats showed reductions in kidney epoxygenase activity and urinary EET excretion (19). Moreover, studies with isolated rabbit collecting ducts (CDs) suggested a role for the EETs and the P450 epoxygenases in distal sodium excretion $(8,9,20)$ and pointed to $\mathrm{ENaC}$ as their molecular target (21). Amiloride and its analogs promote sodium excretion and potassium sparing by selective inhibition of $\mathrm{ENaC}$ (22). To determine the contribution of $\mathrm{ENaC}$ dysfunction to the salt-sensitive Cyp4a10-- hypertensive phenotype,

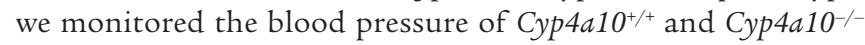
mice on normal salt diets daily, before and after the sequential administration in the drinking water of either amiloride, amiloride in $2 \% \mathrm{NaCl}$, or $2 \% \mathrm{NaCl}$ alone. Within days of administration, amiloride normalized the blood pressure of hypertensive Cyp4a10-/- mice to levels comparable to those of wild-type mice (Figure 2). Cyp $4 a 10^{-/-}$mice on amiloride remained normotensive, even when administered $2 \%$ salt water (Figure 2). Importantly, the effects of amiloride on Cyp4a10-- mice were reversible since, upon its removal, the hypertensive phenotype of the Cyp4a10-/ animals was fully restored (Figure 2). In contrast, amiloride alone or in combination with $2 \% \mathrm{NaCl}$ caused minor changes in the blood pressure of Cyp4a10 ${ }^{+/+}$mice (Figure 2). These results indicate that $\mathrm{ENaC}$ dysfunction accounts for the Cyp4a10/-hypertensive phenotype and suggest that products of the P450 AA monooxygenases could participate in the regulation of $\mathrm{ENaC}$ gating activity.
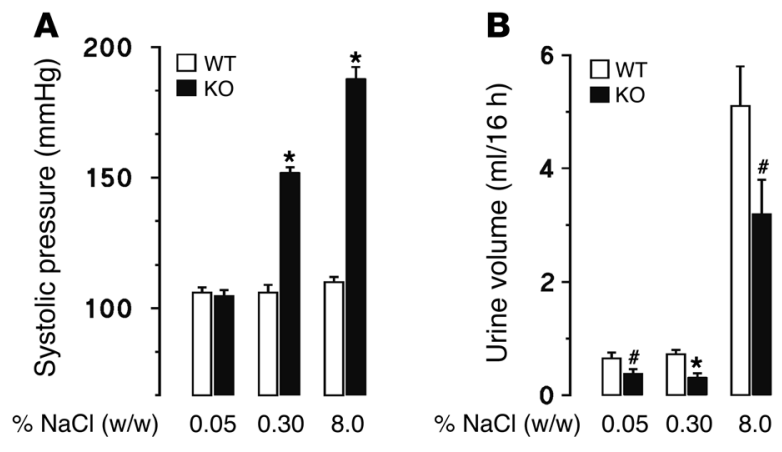

\section{Figure 1}

Hypertension in Cyp4a10-/- mice is salt sensitive. Groups of male Cyp4a10+/+ (WT) (white bars) and Cyp4a10-/- (KO) (black bars) mice were fed low-salt, normal, or high-salt diets (containing $0.05,0.3$, or $8 \%$ $\mathrm{NaCl} \mathrm{w} / \mathrm{w}$ ), respectively. After 3 weeks, their systolic blood pressure (in $\mathrm{mmHg})(\mathbf{A})$ and their urine output during 16-hour collections (B) were measured. Values are averages \pm SEM calculated from at least 14 (low salt), 15 (normal salt), and 24 (high salt) mice. ${ }^{*} P<0.05$; ${ }^{*} P<0.001$, significantly different from wild type. The blood pressure of Cyp4a $10^{+/+}$ and Cyp4a10-/- mice on low-salt diets was not significantly different. 


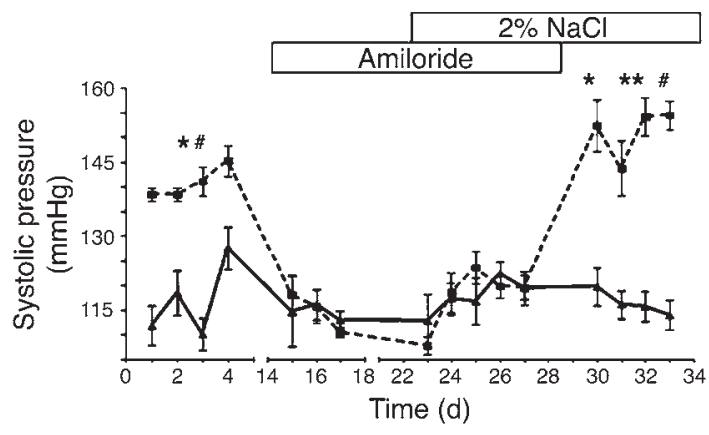

Figure 2

Amiloride normalizes the blood pressure of hypertensive Cyp4a10-/mice. The systolic blood pressure of male Cyp4a10-/- (discontinuous line) and Cyp4a10 ${ }^{+/+}$(continuous line) mice ( $n=5$ each) on diets containing $0.3 \% \mathrm{NaCl}(\mathrm{w} / \mathrm{w})$ were monitored before (days 1 to 4 ) and after the sequential administration of drinking water containing amiloride (days 8 to 21), a mixture of amiloride and $2 \% \mathrm{NaCl}$ (days 22 to 28), and finally $2 \% \mathrm{NaCl}$ alone (days 29 to 34). ${ }^{*} P<0.001$ Cyp4a10-- versus amiloridetreated Cyp4a10 $10^{-/}$mice. ${ }^{\#} P \leq 0.001$ Cyp4a10-/- versus Cyp4a10+/+ mice for all additional amiloride treatment regimes. ${ }^{* \star} P<0.001$ Cyp4a10 ${ }^{-/-}$ mice on water versus Cyp4a10 $10^{-1-}$ mice on $2 \%$ salt water.

Cy4a10-- bypertension in mice is associated with changes in ENaC activity. A recent report (21) provided an explanation for the reported transport effects of the EETs in the distal nephron (8-11) by showing that in rat cortical CDs, (a) AA and 11,12-EET, an epoxygenase metabolite, inhibit ENaC activity; (b) AA-elicited inhibition of $\mathrm{ENaC}$ requires its conversion to EETs; (c) 11,12-EET is formed by $\mathrm{CDs}$, and the CYP2C23 epoxygenase is present in this segment; and (d) $\mathrm{ENaC}$ inactivation by 11,12 -EET is unique since indomethacin, a cyclooxygenase inhibitor, had no effect on the AA-induced responses, nor did 20-HETE mimic the EET response. We therefore studied the effects of AA and of 11,12-EET on the ENaC activity of cortical

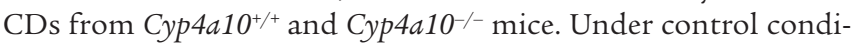
tions, we observed significant differences in $\mathrm{ENaC}$ basal activity between $\mathrm{Cyp} 4 \mathrm{a} 10^{+/+}$(channel number multiplied by the open probability $[\mathrm{NPo}]=0.48)$ and $\mathrm{Cyp}_{4} \mathrm{a} 10^{-/-}(\mathrm{NPo}=1.25)$ mice (Figure 3, $\mathrm{A}$ and $\mathrm{B}$ ). Thus, disruption of Cyp4a10 caused constitutive $\mathrm{ENaC}$ activation and increased sodium reabsorption (Figure $3 \mathrm{~A})$. This is similar to what is observed in Liddle syndrome, a Mendelian form of human hypertension in which mutations in the $\beta$ and $\gamma$ subunits of ENaC cause gain of function and increased sodium currents $(3-7,23)$. Furthermore, while AA effectively inhibited $\mathrm{ENaC}$ gating in wild-type mice (Figure $3, \mathrm{~B}$ and $\mathrm{C}$ ), it had only marginal effects on the knockout mice (Figure 3, A and C), suggesting that these animals had impaired epoxygenase activity. A role for the EETs and the AA epoxygenase in regulating murine ENaC activity was indicated by the demonstration that 11,12-EET inhibits the channel, regardless of Cyp4a10 genotype (Figure 3). In summary, the results shown in Figure 3 provide a mechanistic explanation for the salt-sensitive nature of the Cyp4a10-/- hypertensive phenotype, extend the roles postulated for 11,12-EET in regulating sodium excretion to the murine nephron, and document a functional role for the AA monooxygenases in sodium plasma homeostasis. Thus, genetically determined (Cyp4a10 disruption) reductions in EET biosynthesis or selective epoxygenase inhibition (Figure 3C) (21) equally abrogate the AA-mediated inactivation of $\mathrm{ENaC}$ and result in increased inward sodium flux.
Cy4a10-/- bypertension in mice is associated with changes in renal EETs. To explore the role of the EETs in the in vivo regulation of $\mathrm{ENaC}$ activity and in the Cyp4a10 $10^{--}$hypertensive phenotype, we first measured by mass spectral methods (24) the EETs present endogenously in the kidneys or in urine collected from Cyp4a10 $10^{+/+}$and Cyp4a10 $10^{-/}$mice. While no major differences were observed in EET organ levels, the Cyp4a10-/- mice showed significant reductions in the total amounts of epoxygenase products excreted into their urine during a 16-hour collection period ( $5.1 \pm 0.7$ and $3.2 \pm 0.6 \mathrm{ng}$ for wild-type and knockout mice, respectively; $n=4)$. These results, qualitatively similar to what has been reported for hypertensive Dahl salt-sensitive rats (19), suggested that a reduced renal EET biosynthetic capacity could be responsible for the $\mathrm{ENaC}$ dysfunction observed in hypertensive Cyp4a10-/- mice (Figure 3).

It is now well established that the AA epoxygenases belong to the cytochrome P450 2 gene family (8) and that members of the Cy2c and Cyp2j gene subfamily are expressed in the mouse kidney (25-27). Extensive chemical, biochemical, and immunological evidence show that CYP2C23 is (a) the predominant epoxygenase present in the rat kidney $(28,29)$, (b) the $\mathrm{P} 450$ isoform responsible for the regioand stereochemical properties of the EETs generated by the kidney microsomal proteins $(28,29)$, (c) the renal epoxygenase induced in response to dietary salt loading (29), and (d) the CYP2C epoxygenase expressed in the rat CD (21). From a cDNA library, we cloned and expressed Cyp2c44, the mouse homologue of CYP2C23 (28) (88\% amino acid sequence identity) and demonstrated that purified recombinant Cyp2c44 metabolizes AA to 11,12-EET as its major product ( $65 \%$ of total), with stereoselectivities similar to those previously reported for CYP2C23 $(28,29)$ and Cyp2c44 (27). However, a

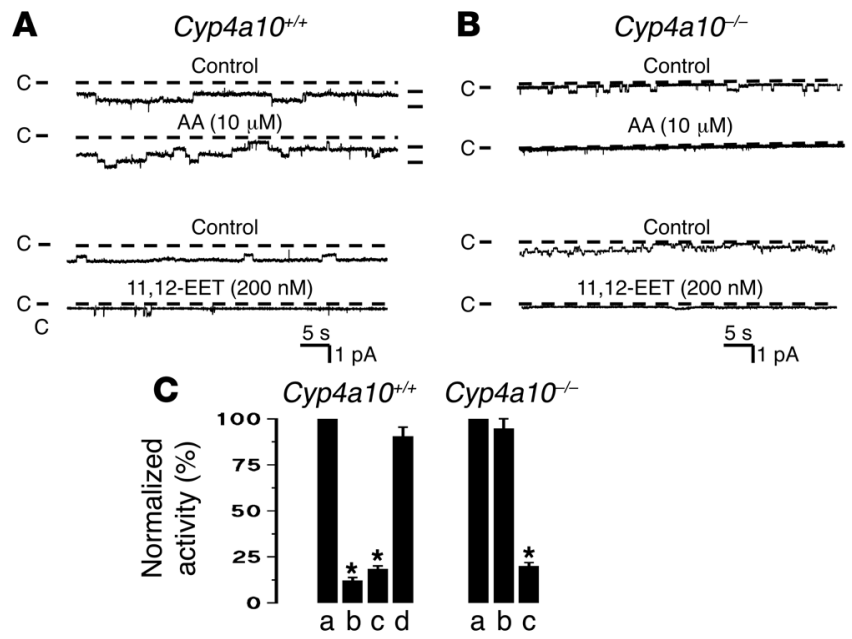

\section{Figure 3}

Effects of AA and 11,12-EET on the ENaC activity of Cyp4a10-/- and Cyp4a10 $10^{+/+}$mice. (A and B) ENaC sodium currents were determined using CD cell attached patches (21). Inward sodium currents when $\mathrm{ENaCs}$ are in the closed state are indicated by $\mathrm{C}$. Short bars indicate channel current levels and dotted lines the current levels for a fully closed channel. (C) Normalized ENaC activities under control conditions (a) or in the presence of either $10 \mu \mathrm{M} \mathrm{AA}$ (b), $200 \mathrm{nM}$ 11,12-EET (c), or a combination of AA and the epoxygenase inhibitor MS-PPOH [N-methylsulfonyl-6-(2-propargyloxyphenyl)hexanamide] (21) (10 and $15 \mu \mathrm{M}$, respectively) (d). Values (as percentage of control activity) are averages \pm SEM calculated from at least 5 different experiments. ${ }^{*} P<0.01$, significantly different from controls. 
Table 2

Wy increases the urinary excretion of epoxygenase metabolites

\begin{tabular}{|c|c|c|c|c|c|}
\hline \multirow[b]{2}{*}{ Urine source } & \multirow[b]{2}{*}{ Treatment } & \multicolumn{4}{|c|}{ Epoxygenase metabolites (EETs and DHETs) ${ }^{\mathrm{A}}$} \\
\hline & & $8,9-$ & $11,12-$ & $14,15-$ & Total \\
\hline Cyp4a10+/+ & None & $1.04 \pm 0.10$ & $0.42 \pm 0.03$ & $0.97 \pm 0.10$ & 2.43 \\
\hline Cyp4a10+/+ & Wy & $1.25 \pm 0.30$ & $0.93 \pm 0.36^{B}$ & $1.30 \pm 0.28$ & 3.48 \\
\hline Cyp4a10-- & None & $0.42 \pm 0.03$ & $0.34 \pm 0.07$ & $0.84 \pm 0.16$ & 1.60 \\
\hline Cyp4a10-- & Wy & $1.12 \pm 0.22^{C}$ & $0.78 \pm 0.09 \mathrm{C}$ & $1.41 \pm 0.11^{\mathrm{D}}$ & 3.31 \\
\hline
\end{tabular}

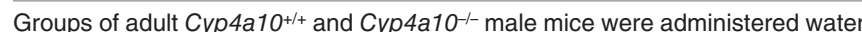
or a solution containing $\mathrm{Wy}(0.02 \% \mathrm{w} / \mathrm{v})$ as drinking water for $7-9$ days. Urine collected from groups of $4-5$ animals was pooled and mixed with synthetic $\left[{ }^{2} \mathrm{H}_{3}\right]$-labeled 8,9-, 11,12-, and 14,15-EET (5 ng each) and [ $\left.{ }^{2} \mathrm{H}_{8}\right]$-labeled 8,9-, $11,12-$, and $14,15-\mathrm{DHET}$ (5 ng each) as internal standards. EETs and DHETs present in the urine samples were extracted, purified, and quantified by LC/MS/MS as described in Methods. Values are averages \pm SEM calculated from at least 4 different experiments. ${ }^{A} \mathrm{ng} / \mathrm{mg}$ of urine creatinine; ${ }^{\mathrm{B} P}<0.04$; ${ }^{C} P<0.019 ; \quad D P<0.008$, significantly different from untreated controls. No significant differences in levels of urinary 8,9 - and 14,15-epoxygenase metabolites were observed between control and Wy-treated Cyp4a10+/+ mice.

study of the effects of dietary salt loading on kidney Cyp2c44 expression by Northern blot or real-time PCR quantification indicated that, while Cyp2c44 was the predominant Cyp2c form expressed in mouse kidney (not shown), salt loading caused variable and limited chang-

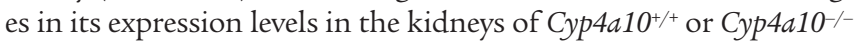
mice. Furthermore, an extended Northern blot and/or real-time PCR study of the effects of salt loading on the levels of Cyp2c29, Cyp2c38, Cyp2c40, Cyp2j5, and Cyp2j6 transcripts, all identified as potential renal AA epoxygenases (25-27), yielded similar results.

To determine whether the experimental manipulation of kidney Cyp2c44 epoxygenase levels and EET biosynthesis normalizes the blood pressure of Cyp4a10 $10^{--}$mice and thus whether reductions in $\mathrm{CD}$ epoxygenase activity could be responsible for the hyperten-

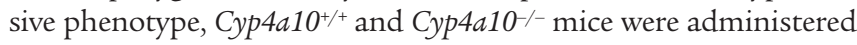
Wyeth 14643 (Wy), a selective PPAR $\alpha$ ligand (30,31), and its effects on kidney epoxygenase metabolite urine excretion, Cyp2c44 mRNA levels, and blood pressure determined. Published studies have shown that PPAR $\alpha$ ligands induce renal CYP2C23 epoxygenase expression (32) and that they reduce blood pressure in rat models of salt-sensitive hypertension $(10,32-34)$. To study the effects of Wy on the renal epoxygenase, we measured by liquid chromatography-tandem mass spectroscopy (LC/MS/MS) the levels of urinary EETs and dihydroxyeicosatrienoic acids (DHETs) before and after treating wildtype and Cyp4a10 null mice with the PPAR $\alpha$ ligand. Since DHETs result only from the hydration of precursor EETs, the combined concentrations of EETs and DHETs are a measure of renal epoxygenase biosynthetic capacity and urinary excretion. In Cyp4a10 $10^{+/+}$mice, Wy treatment caused a 2.2-fold increase in the urinary excretion of the AA 11,12-epoxygenase metabolites (Table 2) and had a more limited impact on 8,9-and 14,15-epoxygenase metabolite excretion (Table 2). On the other hand, and while the overall activity of the renal AA epoxygenase of Cyp4a10 ${ }^{-/-}$mice is lower than that of wild type (Table 2), Wy administration raised the overall excretion levels of epoxygenase metabolites by 2.0 -fold and increased their concentrations to levels similar to those found in the urine of wild-type animals (Table 2). Furthermore, and as with wild-type mice, Wy was most effective in increasing the renal biosynthesis of 11,12-EET (Table 2), the EET regioisomer shown to be the most powerful regulator of $\mathrm{ENaC}$ gating activity (21). As shown in Table 2 and consistent with the

reported regioselectivity of the Cyp2c44 epoxygenase (27), treatment of Cyp4a10-1- mice with Wy also increased the urine levels of the 8,9- and 14,15-AA epoxygenases (2.3- and 1.6-fold increases, respectively) (Table 2). Finally, the levels of epoxygenase metabolites present in the urine provide an averaging measure of overall renal EET biosynthesis and are of limited value in predicting the EET biosynthetic capacity of individual nephron segments. However, studies in dissected rat kidney CDs have demonstrated EET biosynthesis and CYP2C23 epoxygenase expression in this segment (21). The detection sensitivity of current mass spectrometric methods precludes the analysis of EET biosynthesis in mouse nephron segments such as the $\mathrm{CD}$, where $\mathrm{ENaC}$ activity is regulated by 11,12-EET (Figure 3).

In parallel with the increases in epoxygenase metabolites shown in Table 2, Wy treatment raised the concentrations of Cyp2c44 mRNA transcripts present in the Cyp4a10 $0^{+/+}$kidney (Figure 4, A and C) and caused small but significant decreases in the animals' blood pressure (Figure 4B). On the other hand, the PPAR $\alpha$ ligand effectively normalized the blood pressure of hypertensive Cyp4a10 $10^{--}$mice (Figure 4B) and raised their urine epoxygenase metabolites and kidney Cyp2c44 mRNAs to levels similar to or higher than those present in wild-type mice (Table 2) (Figure 4, A and C). This association between an increase in renal Cyp2c44 expression and activity and a reduction in systemic blood pressure supports the antihypertensive functions proposed for the EETs (8-11) and points to the EETs and the AA epoxygenase as endogenous regulators of $\mathrm{ENaC}$ activity $(20,21)$. The mechanism(s) by which the Wy regulates renal Cyp2c44 epoxygenase expression

A

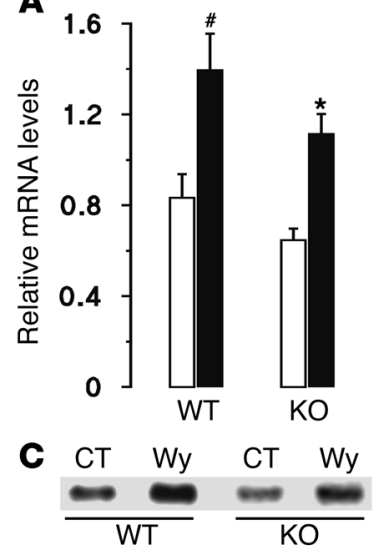

Figure 4

Wy normalizes blood pressure and Cyp2c44 expression in Cyp4a10-/mice. Male Cyp4a10+/+ (WT) and Cyp4a10-/- $(\mathrm{KO})$ mice were administered water (white bars) or a $0.02 \%$ solution of Wy (black bars) as drinking water, and after 10 days, systolic pressure and kidney Cyp2c44 expression were measured. (A) Real-time PCR quantification of Cyp2c44 mRNA levels normalized using the $\beta$-actin mRNA as reference. Shown are averages \pm SEM calculated from 6 experiments. ${ }^{\star} P<0.0005$; $\# P<0.003$, significantly different from untreated controls. (B) Systolic blood pressure (in $\mathrm{mmHg}$ ). Values are averages from groups of 5 mice each. ${ }^{\star \star} P<0.001$, significantly different from untreated knockout; $\# P<0.01$, significantly different from untreated wild type. (C) mRNAs from control (CT) and Wy-treated (Wy) wild-type and knockout (KO) mouse kidneys were analyzed by Northern blot using a Cyp2c44specific probe. Loadings were normalized using a $\beta$-actin probe. 


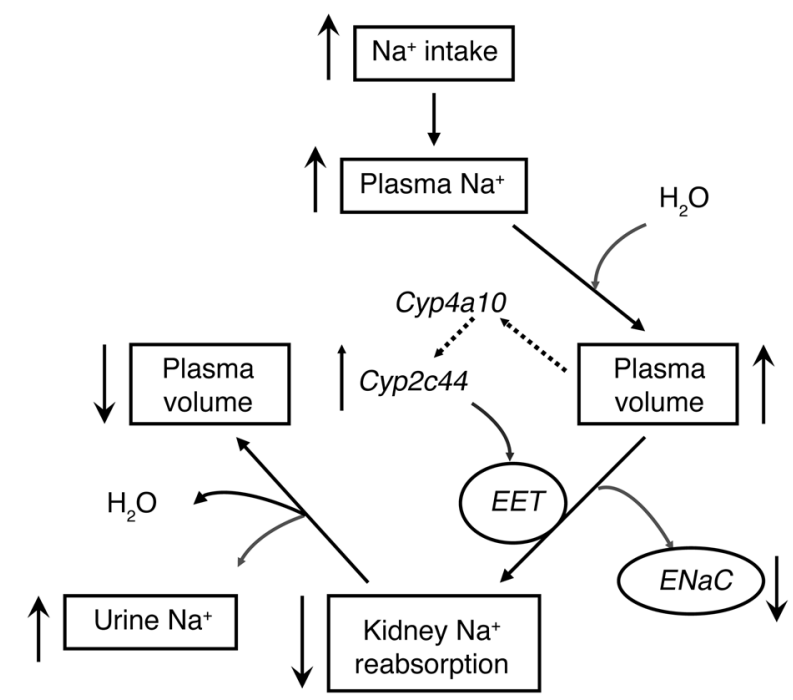

Figure 5

Roles proposed for the epoxygenase regulation of renal sodium reabsorption and plasma volume. Ascending and descending arrows denote increases or decreases in boxed parameters, respectively. Dashed arrows are used to indicate the participation of Cyp4a10 gene product(s) in the regulation of kidney Cyp2c44 levels.

are under study. Nonetheless, the parallel upregulation by Wy of the kidney acyl-CoA oxygenase gene (not shown), a known PPAR $\alpha$ target $(30,31,35)$, suggests a role for this nuclear receptor in the process.

\section{Discussion}

The studies summarized above provide unequivocal evidence of a physiological role for the P450 AA monooxygenases and for the Cyp4a10 gene in particular in the control of $\mathrm{ENaC}$ function, renal $\mathrm{Na}^{+}$transport, and, ultimately, systemic blood pressure. The $\mathrm{Wy}^{-}$ and amiloride-sensitive nature of the Cyp4a10-/- hypertensive phenotype, and the documented association of the pressure effects of these compounds with an upregulated AA epoxygenase expression or $\mathrm{ENaC}$ inhibition, point to a cause-effect relationship among a Cyp4a10-mediated regulation of CD epoxygenase levels, EET biosynthesis, and $\mathrm{ENaC}$ activity. While the mechanism by which products of the Cyp4a10 gene regulate renal epoxygenase expression remains to be defined, there is an established role for PPAR $\alpha$ and its synthetic ligands in the control of P450 expression (30) and of fatty acid metabolism and lipolysis (31). Recent studies have identified several antihypertensive and cardiovascular protective effects resulting from PPAR $\alpha$ activation and/or the administration of synthetic PPAR $\alpha$ ligands (10, 32-34). Furthermore, it has been reported that some of the hypotensive effects of PPAR $\alpha$ ligands are associated with changes in the renal expression of CYP4A (10) or CYP2C (32) isoforms, which are known targets for PPAR $\alpha$-dependent transcriptional control $(30,31)$, and concerted AA oxidation by CYP4A and CYP2C isoforms generates AA epoxy alcohols that are high-affinity PPAR $\alpha$ ligands (35), suggesting that a regulatory loop involving these $\mathrm{P} 450$ isoforms may be involved in endogenous PPAR $\alpha$ activation (35). As an extension of the studies discussed, we are proposing that a dysfunctional Cyp $4 a 10$ gene alters PPAR $\alpha$ ligand biosynthesis and that this impairs the PPAR $\alpha$-dependent expression of a CD Cyp2c epoxygenase and results in reduced CD EET synthesis and increased $\mathrm{ENaC}$ gating.
The CD contributes to the fine tuning of plasma sodium concentrations, and sodium reabsorption by the $\mathrm{CD} \mathrm{ENaC}$ is a rate-limiting step for this physiologically vital function $(2,3,23,36-38)$. Disorders in which gain-of-function mutations in $\mathrm{ENaC}$ cause severe hypertension have illustrated the importance of this channel in the regulation of human blood pressure $(2,3,23,36)$. Like most ion channels, $\mathrm{ENaC}$ function can be regulated by changes in gating properties or membrane density, and the role of aldosterone in regulating apical channel expression in the $\mathrm{CD}$ is well established $(2,3,23,36-38)$. Nonetheless, the early responses to aldosterone occur prior to detectable changes in $\mathrm{ENaC} \mathrm{mRNA}$ or protein levels and involve proteins thought to be responsible for $\mathrm{ENaC}$ trafficking and/or activity, including a serum- and glucocorticoid-inducible kinase (SGK), Nedd4-2, K-ras2, and channel-activating protease 1 (CAP-1) (36-40). Other than these hormone-mediated changes, little is known regarding factors governing intrinsic $\mathrm{ENaC}$ gating activity and sodium currents except that an inositol phospholipid-binding regulatory region in the $\gamma$ subunit of $\mathrm{ENaC}$ was recently identified and characterized (41). The nearly instantaneous nature of the EET effects on ENaC activity (Figure 3, A and B) suggests a role for these eicosanoids in the real-time regulation of this channel and explains their reported effects on sodium transport $(20,21)$.

The phenotypic characterization of Cyp $4 a 10^{-/-}$mice and that reported for Cyp4a14-- mice (15) illustrate the key roles that these 2 genes have in the regulation of tubular function (Cyp4a10) and renal hemodynamics (Cyp4a14) (14) and provide a molecular basis for future studies of the pro- and antihypertensive effects associated with 20-HETE and EETs (9-11), respectively. It is of particular interest that both murine Cyp4a genes exert these renal and pressure effects by controlling the transcriptional activation of alternate P450 genes coding for either 20-HETE or EET synthases.

Based on the results discussed, we propose that under conditions of balanced salt intake and excretion, locally generated EETs participate in nonstimulated $\mathrm{ENaC}$ gating and the regulation of distal sodium reabsorption. Dietary-induced increases in plasma sodium cause compensatory volume changes and rapid and delayed epoxygenase-mediated responses (Figure 5). The rapid phase involves phospholipasedependent AA release and metabolism to EETs. The delayed phase requires an upregulated AA epoxygenase expression and increased EET synthase activity in the CDs (Figure 5). Augmented EET levels cause an EET-mediated inactivation of ENaC, a reduction in inward sodium transport, and increased sodium excretion (Figure 5). Reductions in epoxygenase expression or activity increase ENaCdependent sodium reabsorption and, to maintain plasma sodium levels within physiologically compatible levels, there is increased water retention and expansion of the plasma volume. These epoxygenase-mediated increases in $\mathrm{ENaC}$-dependent sodium reabsorption and the attendant changes in plasma volume result in increased systemic blood pressure (Figure 5) and, ultimately, hypertension. The proposal in Figure 5 is consistent with the normotensive effects of amiloride and Wy on Cyp4a10-/ mice as well as the $\mathrm{ENaC}$ normalizing effect that 11,12-EET has on the CDs of these animals.

The demonstrations of altered tubular transport in salt-sensitive hypertensive Cyp4a10/- mice offer new insights into the mechanisms by which the kidney regulates sodium excretion and suggest that interventions resulting in increased kidney epoxygenase expression (32) or EET concentrations $(42,43)$ could serve as the basis for the development of future antihypertensive therapies. Since an important subset of human hypertensives are salt sensitive (1-5) and this has been associated with alterations in sodium excretion (2-5), we 
propose the human homologues of Cyp4a10 as candidate genes for studies of their role in hypertension. Recent clinical data indicating a role for 20-HETE in human salt-sensitive hypertension (44) and the identification of an association between a human CYP4A11 gene variant and essential hypertension $(45,46)$ lend further support to this proposal. Finally, synthetic PPAR $\alpha$ ligands have been utilized as hypolipidemic agents with limited side effects. Based on the studies reported here and elsewhere (32-34), the evaluation of their potential antihypertensive effects in humans seems warranted.

\section{Methods}

cDNA cloning, expression, and enzymatic analysis. The Cyp4a10, 4a12, 4a14 and $2 \mathrm{c} 44$ cDNAs were cloned and expressed in E. coli $(14,16,47)$ (the Cyp4a10 gene sequence is available at www.ncbi.nih.gov; MGI:88611, 13117). Northern blot analyses of RNAs isolated from the kidneys of Cyp4a10-/- mice revealed the presence of transcripts recognized by the Cyp4a 10 probe in these animals. RT-PCR cloning using Cyp4a10 forward (5'-TTGCAGACAGCCTCTCTGGCTTCC- $\left.3^{\prime}\right)$ and reverse primers (5'-CTCGAGGTTACAGACTCTTCTC- ${ }^{\prime}$ ) and sequence analysis showed that these RNA transcripts originated from a Cyp4a10 pseudogene and that, compared with the Cyp4a10 mRNA, they contained several single nucleotide replacements, a $429 \mathrm{bp}$ intronic insertion, and a 17 bp deletion at nucleotide 1255 (from the ATG translation initiator) that causes a frame shift and would generate a protein lacking a heme prosthetic group.

mRNA expression levels were estimated by Northern blot (15) or real-time quantitative PCR using a Bio-Rad iCycler iQ instrument (Bio-Rad) and the manufacturer's reagents and software (Gene Expression Analysis for iCycler iQ Real Time PCR Detection System). Total RNAs were isolated from whole mouse kidneys using the TRIzol Reagent (Invitrogen Corp.) and, in some experiments, purified by oligo(dT) chromatography. For real-time quantitative PCR, kidney reverse transcriptase products were amplified using the following primer pairs: Cyps 2b9, 2b10, and 2b19: 5'-TGCTCCACGAGACTACATTGA-3' and 5'-GTACTTGAGCATGAGCAGGA-3'; Cyp2c29: 5'-TGTCCTGGAAGTCATCACAAA- $3^{\prime}$ and $5^{\prime}$-TTGTTCAATGTGGTTTACCTGTT-3'; 2c37: $5^{\prime}$-CTGCTTTCCACACTGGACAC-3' and $5^{\prime}$-CAGGTCCATATCATCACAGCA-3'; Cyp2c38: 5'-CCCCCAGGTTTTTATTCACC-3' and 5'-GCATAAGGGCTCAGACAACTG-3'; 2c39: 5'-CCTCCTTTATTGCCTTCTGC-3' and 5'-TGCATTTGTGCTATAACTCCAGA-3'; Cyp2c40: 5'-CATGGATATGACCCCCAAAC-3' and 5'-TGGGACTGGGTTAAATGAGG-3'; Cyp2c44: 5'-TGCTACCTAGGCCAGAAACA-3' and $5^{\prime}$-GATGAGGGCCTTGTGGATCT-3'; Cyp2j5: 5'-CCTGCTCACATGAGAAGTGATAG-3' and 5'-GATGATAAGGTATGTCACTGGACAC-3'; Cyp2j6: 5'-GGTTGCTCACACGAGAGAAG-3' and 5'-GCATGCCACTGAGCTCATAA-3'; $\beta$-actin: $5^{\prime}$ AGGTGACAGCATTGCTTCTG-3' and $5^{\prime}$-AGGGAGACCAAAGCCTTCAT$3^{\prime}$. Averaged Сур $2 \mathrm{c}$ and $\beta$-actin fluorescence threshold crossing cycle calculated from a PCR baseline subtracted from curve fit $\left(C_{\tau}\right)$ values were utilized to calculate $\beta$-actin normalized mRNA expression levels.

Kidney microsomes were isolated and incubated with $\left[1-{ }^{14} \mathrm{C}\right] \mathrm{AA}(100 \mu \mathrm{M})$ and NADPH ( $1 \mathrm{mM}$ ) as described (48). Purified recombinant P450s $(0.1 \mu \mathrm{M})$ were incubated with purified rat $\mathrm{P} 450$ reductase $(1 \mu \mathrm{M})$ and dilauroylphosphatidylcholine $(50 \mu \mathrm{g} / \mathrm{ml})$ prior to the addition of AA $(75 \mu \mathrm{M})$ and NADPH $(1 \mathrm{mM})$. Cyp4a proteins were reconstituted in the presence of cytochrome $b_{5}$ $(15,48)(0.1 \mu \mathrm{M})$. Products were quantified as published $(48)$. The creatinine

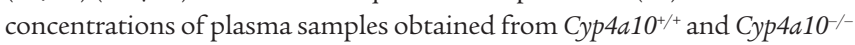
mice fed solid diets containing normal salt $(0.3 \% \mathrm{NaCl} w / w)$ or high salt $(8 \%$ $\mathrm{NaCl}$ w/w for 14-16 weeks) were determined as reported (14).

Construction of the Cyp4a10-targeting vector. Overlapping clones encoding all Cyp4a10 exons were isolated from a 129/SvJ mouse BAC genomic library (Invitrogen Corp.). A linearized pNTK targeting vector containing an Nco 1 flanked Cyp4a10 gene segment in which exon 3 and part of exon 4 were replaced by a neomycin cassette was electroporated into TL-1 129/SvEv Tac mouse ES cells. Recombinant ES cells were identified by Southern blots of Acc 1 digests using a $1.0 \mathrm{~kb}$ Cyp $4 a 10$ probe coding for exon 1 and segments of its flanking intron and $5^{\prime}$ upstream sequences. The neomycin replacement removed exon 3 of the Cyp4a10 gene, interrupted in-frame translation at exon 4, and eliminated a diagnostic AccI site, used for genotype analysis. Two ES clones carrying a Cyp4a10 mutant allele (IE5 and ID12) were utilized for the generation of germ line chimeras. Mice were fed commercial solid diets containing $0.05,0.3$, or $8 \% \mathrm{NaCl}(\mathrm{w} / \mathrm{w})$ and allowed free access to water. Amiloride was administered in the drinking water and its concentrations adjusted to a daily dose of approximately $2 \mathrm{mg} / \mathrm{kg}$ of body weight. Wy was administered as a $0.02 \% \mathrm{w} / \mathrm{v}$ solution in the drinking water. Animal experiments were performed in accordance with NIH policies and approved by the Vanderbilt University Medical Center Institutional Animal Care and Use Committee.

Blood pressure measurements. Systolic blood pressure of conscious 12- to 20-week-old mice was measured at an ambient temperature of $23^{\circ} \mathrm{C}$ using a tail cuff blood pressure analyzer (model 178; IITC Inc.). Prior to measurement, animals were exposed to the environment and instrument on a daily basis for at least 7 days. The blood pressure of trained mice was monitored for 30-40 minutes and final values obtained after 4 consecutive readings whose values were within $\pm 5 \%$ of the mean. In some experiments, blood pressure was measured by means of a Micro-Renthane tapered catheter (300-500 $\mu \mathrm{m}$ OD) inserted into the right carotid artery as described (15). After phenotype confirmation with the Cyp4a10-- ID12 clone, all subsequent studies were done using the Cyp4a10/- IE5 line. Daily measurements of free fluid volume (16) were done at $10 \mathrm{am}$ and at $3 \mathrm{pm}$ for 3 consecutive days and in groups of age-matched male Cyp $4 a 10^{+/+}$and Cyp4a10 $10^{--}$mice (at least 8 animals each), using a Bruker Minispec mq10 (Bruker BioSpin Corp.) nuclear magnetic resonance instrument. Urinary sodium and potassium were measured by flame photometry.

Analysis and quantification of urinary epoxygenase products. Overnight urine samples were collected from groups of 4-5 adult mice in flasks containing triphenylphosphine (2-3 mg each) (30). After adding synthetic $\left[20-{ }^{2} \mathrm{H}_{3}\right]$-labeled 8,9-; 11,12-; and 14,15-EET (5 ng each) and [5,6-, 8,9-, 11,12-, and 14,15- $\left.{ }^{2} \mathrm{H}_{8}\right]-$ labeled 8,9-; 11,12-; and 14,15-DHET (5 ng each) as internal standards, the urine EETs and DHETs were extracted with acidified $\mathrm{CHCl}_{3} / \mathrm{CH}_{3} \mathrm{OH}(2: 1)$ (24), purified by reverse phase HPLC as described (24), and methylated by reaction with diazomethane in ethyl ether (24). The resulting methyl-EETs and methyl-DHETs were quantified by LC/MS/MS using an Agilent Eclipse XDB-C 8 column $(2.1 \times 150 \mathrm{~mm} ; 5 \mu \mathrm{m})$ connected to a TSQ-Quantum MS/MS spectrometer (Thermo Electron Corp.). EETs and DHETS were resolved using a linear solvent gradient that went from $80 \% \mathrm{CH}_{3} \mathrm{OH}, 20 \% \mathrm{H}_{2} \mathrm{O}$ containing $25 \mu \mathrm{M} \mathrm{AgBF}_{4}$ to $100 \% \mathrm{CH}_{3} \mathrm{OH}$ containing $25 \mu \mathrm{M} \mathrm{AgBF}_{4}$ in over 10 minutes and at a flow of $0.2 \mathrm{ml} / \mathrm{min}$. For analysis and quantification, we utilized collision-induced fragmentation of the methyl-EET ${ }^{107} \mathrm{Ag}$ adducts at $\mathrm{m} / \mathrm{z} 441$ and 444 (corresponding to the molecular ions of the biological sample and the internal standard, respectively) and of the methyl-DHET ${ }^{107} \mathrm{Ag}$ adducts at $\mathrm{m} / \mathrm{z} 459$ and 467 (corresponding to the molecular ions of the biological and the internal standard, respectively). Diagnostic selective product ion analysis was done at $\mathrm{m} / \mathrm{z} 261,247$, and 287 for ${ }^{107} \mathrm{Ag}$ adducts of the methyl esters of 8,9-, 11,12-, and 14,15-EET, respectively; at $\mathrm{m} / \mathrm{z} 317,34,7$ and 329 for the ion fragments generated by the ${ }^{107} \mathrm{AG}$ adducts of the methyl esters of 8,9-, 11,12-, and 14,15-DHET, respectively; and at $\mathrm{m} / \mathrm{z} 323,353$, and 335 , respectively, corresponding to the ion fragments originating from the corresponding ${ }^{107} \mathrm{Ag}$ adducts of the deuterated internal standards. Quantifications were done using isotope ratios and ion intensities versus mass calibration curves (24).

$\mathrm{CD}$ isolation and electrophysiology. Male wild-type and Cyp4a10-/- mice were fed low-sodium diets for 5-7 days. Cortical CDs microdissected from kidney slices ( $1 \mathrm{~mm}$ thickness) were placed on an inverted microscope, superfused with HEPES-buffered $\mathrm{NaCl}$ (21), and cut open to expose the apical membrane. Sodium currents were recorded and digitized as described (21). 
Channel activity, defined as NPo, was calculated from data samples of 60 seconds during steady state as follows: NPo $=\Sigma\left(t_{1}+2 t_{2}+\ldots+i t_{i}\right)(20)$, where $t_{i}$ is the fractional open time spent at each observed current level. Channel conductance was calculated from the current intensities recorded at 3 or more holding potentials.

Statistics. Statistical analyses were performed using a 2-tailed Student's $t$ test. $P<0.05$ was considered statistically significant.

\section{Acknowledgments}

We are grateful to Alberto Nasjletti and Eric Johnson for valuable discussions and suggestions and to the NIH (grants DK38226 and HL 34300 to W.-H. Wang and Y. Wei) and the Robert A. Welch Foundation (J.R. Falck) for their generous support. The contribu-

tions of the Vanderbilt University Small Animal Physiology Core to these studies are also gratefully acknowledged.

Received for publication December 1, 2005, and accepted in revised form March 7, 2006.

Kiyoshi Nakagawa and Vijaykumar R. Holla contributed equally to this work.

Address correspondence to: Jorge H. Capdevila, Vanderbilt University Medical Center North S-3223, Nashville, Tennessee 37232, USA. Phone: (615) 322-4968; Fax: (615) 343-4704; E-mail: jorge. capdevila@vanderbilt.edu.

1. Chaobanian, A.V., et al. 2003. Seventh report of the Joint National Committee on Prevention, Detection, Evaluation, and Treatment of High Blood Pressure. Hypertension. 42:1206-1252.

2. Guyton, A.C. 1991. Blood pressure control: special role of the kidneys and body fluids. Science. 252:1813-1816.

3. Lifton, R.P., Gharavi, A.G., and Geller, D.S. 2001. Molecular mechanisms of human hypertension. Cell. 104:545-556.

4. Strazzullo, P., Galletti, F., and Barba, G. 2003. Altered renal handling of sodium in human hypertension. Short review of the evidence. Hypertension. 41:1000-1005.

5. Shaughnessy, K.M., and Karet, F.E. 2004. Salt handling and hypertension. J. Clin. Invest. 113:1075-1081. doi:10.1172/JCI200421560.

6. Glueck, S.B., and Dzau, V.J. 2002. Physiological genomics:implications in hypertension research. Hypertension. 39:310-315.

7. Luft, F.C. 2004. Present status of genetic mechanisms in hypertension. Med. Clin. North Am. 88:1-18.

8. Capdevila, J.H., and Falck, J.R. 2002. Biochemical and molecular properties of the cytochrome P450 arachidonic acid monooxygenases. Prostaglandins Other Lipid Mediat.68-69:325-344

9. McGiff,J.C., and Quilley,J. 2001.20-Hydroxyeicosatetraenoic acid and epoxyeicosatrienoic acids and blood pressure. Curr. Opin. Nephrol. Hypertens. 10:231-237.

10. Roman, R.J. 2002. P450 metabolites of arachidonic acid in the control of cardiovascular function. Physiol. Rev. 82:131-185.

11. Zhao, X., and Imig, J.D. 2003. Kidney CYP450 enzymes: biological actions beyond drug metabolism. Curr. Drug Metab. 4:73-84.

12. Nabika, T., Kobayashi, Y., and Yamori, Y. 2000. Congenic rats for hypertension: how useful are they for the hunting of hypertension genes? Clin. Exp. Pharmacol. Physiol. 27:251-256.

13. Garret, M.R., Saad, Y., Dene, H., and Rapp, J.P. 2000. Blood pressure QTL that differentiate Dahl salt-sensitive and spontaneous hypertensive rats. Physiol. Genomics. 29:33-38.

14. Dunn, S.R., Qi, Z., Bottinger, E.P., Breyer, M.D., and Sharma, K. 2004. Utility of endogenous creatinine clearance as a measure of renal function in mice. Kidney Int. 65:1959-1967.

15. Holla, V., et al. 2001. Alterations in the regulation of androgen-sensitive Cyp 4a monooxygenases cause hypertension. Proc. Natl. Acad. Sci. U. S. A. 98:5211-5216.

16. Hockings, P.D., and Rogers, P.J. 1994. 1H NMR determination of intracellular volume in cell suspensions. Arch. Biochem. Biophys. 311:383-388.

17. Heng, Y.M., et al. 1997. A novel murine gene, Cyp4a14, is part of a cluster of Cyp4a and 4b, but not Cyp4F genes in mouse and humans. Biochem. J. 325:741-749.

18. Capdevila, J.H., et al. 1992. Cytochrome P-450 arachidonic acid epoxygenase. Regulatory control of the renal epoxygenase by dietary salt loading.
J. Biol. Chem. 267:21720-21726.

19. Makita, K., et al. 1994. Experimental and/or genetically controlled alterations of the renal microsomal cytochrome $\mathrm{P} 450$ epoxygenase induce hypertension in rats fed a high salt diet. J. Clin. Invest. 94:2414-2420.

20. Jacobson, H.R., et al. 1984. Effects of epoxyeicosatrienoic acids on ion transport in the rabbit cortical collecting tubule. In Prostaglandins and membrane ion transport. P. Braquet, J.C. Frolich, S. Nicosia, and R. Garay, editors. Raven Press. New York, New York, USA. 311-318.

21. Wei, Y., et al. 2004. Arachidonic acid inhibits epithelial Na channel via cytochrome P450 (CYP) epoxygenase dependent pathways. J. Gen. Physiol. 124:719-727.

22. Schild, L., Schneeberger, E., Gautschi, I., and Firsov, D. 1997. Identification of amino acid residues in the alpha, beta, and gamma subunits of the epithelial sodium channel $(\mathrm{ENaC})$ involved in amiloride block and ion permeation. J. Gen. Physiol. 109:15-26.

23. Rossier, B.C., Pradervand, S., Schild, L., and Hummler, E. 2002. Epithelial sodium channel and the control of sodium balance: interactions between genetic and environmental factors. Annu. Rev. Physiol. 64:877-897.

24. Capdevila, J.H., Dishman, E., Karara, A., and Falck, J.R. 1991. Cytochrome P450 arachidonic acid epoxygenase: stereochemical characterization of epoxyeicosatrienoic acids. Methods Enzymol. 206:441-453.

25. Ma, J., et al. 1999. Molecular cloning, enzymatic characterization, developmental expression, and cellular localization of a mouse cytochrome P450 highly expresses in kidney. J. Biol. Chem. 274:17777-17788.

26. Tsao, C.C., et al. 2001. Identification and localization of five CYP2Cs in murine extrahepatic tissues and their metabolism of arachidonic acid to regioand stereoselective products. J. Pharmacol. Expt. Ther. 299:39-47.

27. DeLozier, T.C., et al. 2004. CYP2C44, a new murine CYP2C that metabolizes arachidonic acid to unique stereospecific products. J. Pharmacol. Exp. Ther. 310:845-854.

28. Karara, A., et al. 1993. Molecular cloning, expression, and enzymatic characterization of the rat kidney cytochrome P-450 AA epoxygenase. J. Biol. Chem. 268:13565-13570.

29. Holla, V.R., Makita, K., Zaphiropoulos, P.G., and Capdevila, J.H. 1999. The kidney cytochrome P450 $2 \mathrm{C} 23$ epoxygenase is upregulated during dietary salt loading. J. Clin. Invest. 104:751-760.

30. Savas, U., Griffin, K.J., and Johnson, E.F. 1999. Molecular mechanisms of cytochrome P450 induction by xenobiotics: an expanded role for nuclear hormone receptors. Mol. Pharmacol. 56:651-857.

31. Mandard, S., Muller, M., and Kersten, S. 2005. Peroxisome proliferator-activated receptor alpha target genes. Cell. Mol. Life Sci. 61:393-416.

32. Muller, D.N., et al. 2004. A peroxisomal proliferator-activated receptor- $\alpha$ activator induces renal
CYP2C23 activity and protects from angiotensin II-induced renal injury. Am. J. Pathol. 164:521-532.

33. Schiffrin, E.L., Amiri, F., Benkirane, K., Iglarz, M., and Diep, Q.N. 2003. Peroxisomal proliferator activated receptor: vascular and cardiac effects in hypertension. Hypertension. 42:664-668.

34. Semenkovich, C.F. 2004. Fatty acid metabolism and vascular disease. Trends Cardiovasc. Med. 14:72-76.

35. Cowart, L.A., et al. 2002. The CYP 4A isoforms hydroxylate epoxyeicosatrienoic acids to form high affinity PPAR ligands. J. Biol. Chem. 277:35105-35112.

36. Snyder, P.M. 2002. The epithelial $\mathrm{Na}^{+}$channel: cell surface insertion and retrieval in $\mathrm{Na}^{+}$homeostasis and hypertension. Endocr. Rev. 23:258-275.

37. Loffin, J., et al. 2000. Differential subcellular localization of $\mathrm{ENaC}$ subunits in mouse kidney in response to high- and low-Na diets. Am. J. Physiol. Renal Physiol. 279:F252-F258.

38. Loffin, J., et al. 2001. Aldosterone induces rapid apical translocation of $\mathrm{ENaC}$ in early portion of renal collecting system: possible role of SGK. Am. J. Physiol. Renal Physiol. 280:F675-F682.

39. Gormley, K., Dong, Y., and Sagnella, G.A. 2003. Regulation of the epithelial sodium channel by accessory proteins. Biochem. J. 371:1-14.

40. Farjah, M., Roxas, B.P., Geenen, D.L., and Danziger, R.S. 2003.Dietary salt regulates renal SGK1 abundance. Relevance to salt sensitivity in the Dahl rat. Hypertension. 41:874-878.

41. Pochynyuk, O., Staruschenko, A., Tong, Q., Medina, J., and Stockand, J.D. 2005. Identification of a functional phosphatidylinositol 3,4,5-trisphosphate binding site in the epithelial $\mathrm{Na}^{+}$channel. J. Biol. Chem. 280:37565-37571.

42. Yu, Z., et al. 2000. Soluble epoxide hydrolase regulates hydrolysis of vasoactive epoxy-eicosatrienoic acids. Circ. Res. 87:992-998.

43. Sinal, C.J., et al. 2000. Targeted disruption of soluble epoxide hydrolase reveals a role in blood pressure regulation. J. Biol. Chem. 275:40504-40510.

44. Laffer, C.L., Laniado-Schwartzman, M., Wang, M.H., Nasjletti, A., and Elijovich, F. 2003. Differential regulation of natriuresis by 20 -hydroxyeicosatetraenoic acid in human salt-sensitive versus saltresistant hypertension. Circulation. 107:574-578.

45. Gainer, J.V., et al. 2005. A functional variant of CYP4A11 20-hydroxyeicosatetraenoic acid synthase is associated with essential hypertension. Circulation. 111:63-69.

46. Mayer, B., et al. 2005. Association of the T8590C polymorphism of CYP4A11 with hypertension in the MONICA Augsburg echocardiographic substudy. Hypertension. 46:766-771.

47. Barnes, H.J., Arlotto, M.P., and Waterman, M.R. 1991. Expression and enzymatic activity of recombinant cytochrome P450 17 $\alpha$-hydroxylase in Escherichia coli. Proc. Natl. Acad. Sci. U. S. A. 88:5597-5601.

48. Capdevila, H., Falck, J.R., Dishman, E., and Karara, A. 1989. Cytochrome P450 arachidonate oxygenase. Methods Enzymol. 187:385-394. 